\title{
Corrélations hépato-pancréato-biliaires
}

^Association (françaíse) d'Etudes Physío-pathologíques du Foie vient de tenir à Geneve (23/24 avril 1955) la $1^{\circ}$ Session de son XIX0 Congrès consacré au sujet indiqué dans ce titre; et il nous parait intéressant d'exposer les principales conclusions à tirer des remar-quables rapports présentés au cours de 3 seances de travail dense, et des discussions nourries qui ont suivi. Dans son discours inaugural, le president E. Martin (Geneve) a souligné notamment les differences profondes qui existent (comme c'est le cas en pathologie hépatique) entre l'insuffisance pancréatique «pancréas-présent», c'est-à-dire au cours des maladies du pancreas, 19 Gastroenterologia, Vol. 83, No. 4 (1955)

244

Editorial

et celle qui se développe «pancréas-absent», c'est-à-dire consecutive à la pancréatectomie totale. En corrolaire, après avoir envisage, de façon très nuancée, les indications des pancréatectomíes totale ou partielle dont il possède une grande experience personnelle, A. Leaner (Lucerne) décrit les consequences de ces operations et les remèdes à y apporter.

Les auteurs des rapports bíologiques ont insisté surtout sur les correlations entre les parties endocrine et exocrine du pancreas. Dans les diabetes provoqués par Гalloxane ou Гacide dialurique, $\Gamma$ histologiste A. Faller (Fribourg) a démontré la possibilité de transformation des cellules acineuses en cellules insulaires; tandis que le physiologiste A. Loubatíères (Montpellier) passait en revue successivement: le retentissement endocrinien des modifications de la glande à secretion externe; les reactions des cellules acineuses à insuline ou aux diabetes expérimentaux; enfin les influences hormonales susceptibles d'agir simultanément sur les fractions endo- et exocrine du pancreas.

Cela devait amener tout naturellement à parler du diabète des pancréatítes chroníques. Selon Ch. Darnaud (Toulouse), le diabète accompagne au moins une fois sur trois les alterations du pancreas (frequence contestée par A. Vachon, Lyon). Ce diabète demeure bénin, non-acidosique; il est très sensible à $\Gamma$ insuline et évolue par poussées parallèles à $\Gamma$ extension des lesions de la glande, lesions qui confèrent au syndrome des douleurs épigastriques gauches, inhabi-tuelles dans le diabète. Les pancréatites chroniques peuvent d'ail-leurs tout aussi bien s'accompagner d'hypoglycémie, puisque les îlots de Langerhans possèdent, à côté des cellules $\beta$, sécrétrices de Tinsuline hypoglycémiante, des cellules a responsables de la production du glucagon, hormone glycogénolytique et hyperglycémiante. Pour Loubatières, une meilleure connaissance de ces correlations entre les deux functions externe et interne du pancreas devrait induire les cliniciens à reconnaître le pré-diabète, à temps pour prévenir ou retarder Гinstallation du diabète vrai. Les calcifications pancréa-tiques, notamment, peuvent provoquer de la stase dans les canali-cules, où Taugmentation de la pression possède un effet traumatisant sur les îlots.

Dans le domaine de 1'exploration, Гexposé très fouillé de J. Warter (Strasbourg) sur les méthodes de laboratoire destinées à mettre en evidence les lesions du pancreas exocrine, était empreint d'un certain scepticisme, même en ce qui concerne la méthode personnelle du rapporteur: le «transit lipidique», c'est-à-dire Гingestion d'un repas 
Editorial

245

baryté additionné d'huile d'olive, et dosage de la proportion de corps gras restant dans la baryte expulsée avec les selles. H. Staub (Bale), après avoir essayé dans sa clinique de nombreuses techniques nou velles, en est revenu à l'analyse coprologique après repas standard, et au dosage de Tamylasurie (selon Wohlgemuth); A. Vachon lui préfère le dosage dans les urines, de l'iode libéré après digestion d'une huile iodée (épreuve de Trémolières).

IS exploration radiologíque a été étudiée par R. Sarasin (Geneve), qui insiste longuement sur les symptômes duodénaux des pancréa-tites, appuyé en celà par J. Baumel (Montpellier). De son côté L. Léger (Paris) présenta sa technique originale, consistant à ca-thétériser la canal de Wirsung au moyen d'un tube de polythene introduit dans Tampoule de Vater, après duodénotomie. De nombreux et superbes cliches illustrèrent cette pancréatographíe dírecte, montrant les résultats déjà obtenus - et ce n'est qu'un debut - sans danger pour le malade, à condition d'injecter dans le canal de Wirsung des quantités très minimes ( $3 \mathrm{cc}$. au maximum) de substance de contraste. Puis P. Porcher et J. Carolí émerveillèrent les participants avec leur dernière realisation: la ciné-radíomanométríe, grace à laquelle - et à raison de 960 images par minute! - ils projettent sur Гécran tout le detail des mouvements du cholédoque, de la region ampullaire et le reflux wirsungien!

Le rapport intitule «l'état du foie dans les pancréatites chroni-ques» était dévolu à H. Staub (Bale); celui-ci tente d'établir une unite physíopathologíque hépato-pancréatíque à l'aide de nombreux arguments de tout ordre, parmi lesquels il faut retenir:

L'irrigation de deux organes par le même tronc coeliaque, et les repercussions des troubles vasculaires ou neuro-vasculaires à ce niveau, sur le pancreas comme sur le foie.

Les «relations métaboliques nutritivo-enzymatiques», c'est-à-dire le déterminisme d'une stéatose identique du foie, par la carence en substances lipotropiques dans la ration alimentaire, comme par l'insuffisance en hormone lipocaïc du pancreas; A. Froehlích (An-vers) remarque d'ailleurs que cette vérité expérimentale ne possède pas d'expression clinique, la dégénérescence graisseuse du foie ne se retrouvant guère dans les pancréatites chroniques.

L'influence de substances toxiques comme Véthíoníne, capables d'attaquer à la fois les cellules parenchymateuses hépatiques et les acini pancréatiques, technique que M. Coppo et l'école de Modène ont particulièrement étudiée. 